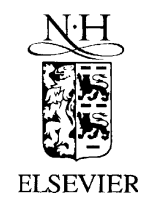

Acta Psychologica 114 (2003) 17-39

\section{acta psychologica}

www.elsevier.com/locate/actpsy

\title{
Catching oriented objects
}

\author{
Frouke Hermens *, Stan Gielen
}

Department of Biophysics, Facultiet der Natuurwetenschappen, University of Nijmegen, Wiskunde en Informatica, Geert Grootteplein 21, 6525 EZ Nijmegen, The Netherlands

Received 22 October 2002; received in revised form 20 April 2003; accepted 20 April 2003

\begin{abstract}
We have investigated how participants match the orientation of a line, which moves on a vertical screen towards the subject. On its path to the participant, the line could disappear at several positions. Participants were instructed to put a bar on a predefined interception point on the screen, such that the bar touched the screen with the same orientation as the moving line at the very moment when the line passed through the interception point or (in case of line disappearance) when the hidden line would pass through the interception point (like in catching). Participants made significant errors for oblique orientations, but not for vertical and horizontal orientations of the moving line. These errors were small or absent when the moving line was visible all the way along its path on the screen. However, these errors became larger when the line disappeared farther away from the interception point. In the second experiment we tested whether these errors could be related to errors in visual perception of line orientation. The results demonstrate that errors in matching of the bar do not correspond to the last perceived orientation of the line, but rather to the perceived orientation of the moving line near the begining of the movement path. This corresponds to earlier observations that participants shortly track a moving target and then make a saccadic eye movement to the interception point.
\end{abstract}

(C) 2003 Elsevier B.V. All rights reserved.

PsycINFO classification: 2323; 2330

Keywords: Catching; Visual parallelism

\footnotetext{
* Corresponding author.

E-mail address: froukeh@mbfys.kun.nl (F. Hermens).
} 


\section{Introduction}

Many studies have stressed the importance of studying action and perception as two connected processes (Berthoz, 1993). A good example is the study of catching a moving object, where vision provides the information about position and orientation of the object to trigger a sequence of muscle activation patterns such that shoulder and elbow movements bring the hand to a location where the object will be just after the movement is completed.

The process of catching a moving object involves many complex subprocesses within the perception-action cycle. One important subprocess of catching movements is the preshaping of the hand (e.g., Gentilucci, 2002; Santello \& Soechting, 1998). In most of the experiments in which the preshaping of the hand during catching movements was studied, participants were asked to catch a spherical object, like a ball (Savelsbergh \& Whiting, 1996). When objects are not spherical but have a clear asymmetry and orientation, catching is more complicated since the orientation of the object in space has to be determined in order to catch the object properly. In this context it should be remarked that participants make systematic errors in matching the orientation of cylindrical bars or lines at different locations visually or haptically (Cuijpers, Kappers, \& Koenderink, 2000; Hermens \& Gielen, 2003; Kappers \& Koenderink, 1999). This raises the question whether these matching errors also affect the preshaping of the hand in catching movements. Of importance here is whether vision for perception and vision for action take place in separate, independent neural pathways (Goodale \& Milner, 1992). If vision for perception and vision for action make use of the same underlying representation, any errors in the percept of object orientation should be reflected in errors in catching movements to these objects.

The question to what extent vision for perception and vision for action are related, has been investigated by comparing the effect of a perceptual illusion on size estimation and grasping. For example, participants were asked to estimate the size of and to grasp the disc inside the Tichener circles illusion or the bar inside the Müller-Lyer illusion. Some studies have found that subjects could grasp objects correctly, even when the size of an object was perceived incorrectly (e.g., Haffenden \& Goodale, 1998; Haffenden, Chif, \& Goodale, 2000). The absence of an effect of the illusion on grasping movements in these studies suggests that vision for perception and vision for action use different neuronal pathways. Later studies have shown that a delay between the offset of the presentation of the stimulus and the onset of the grasping movement causes the peak hand aperture of the grasping movement to depend on the size of the illusion (Hu, Eagleson, \& Goodale, 1999; Hu \& Goodale, 2000; Westwood, McEachern, \& Roy, 2001). The effect of the delay has been explained by assuming that the representation for action decays quickly. After the delay the action representation of the object has decayed and the grasp is planned based on the perceptual representation.

Here we report results of experiments which investigated the effect of visual perception of object orientation on orientation adjustment of the hand in a movement, which resembled a catching movement. In the first experiment participants were asked to match the orientation of a moving line on a vertical screen by bringing a 
hand-held bar on a future position of the moving line in the same orientation as that of the line. They were asked to put the bar on the screen at the very moment in time when the moving line passed through a predefined interception point. This task allowed us to investigate the orientation of the hand when participants would be trying to "catch" the moving line displayed on the screen. On its path towards the participant the line could disappear at one of two possible locations. If it disappeared, it could do so either just before the interception point or halfway in between the starting position and interception point. The question we addressed was whether participants match the orientation of the moving line correctly by the hand-held bar, or whether they made errors corresponding to the errors in visual perception of the orientation of the line at, or before the time of disappearance.

In the first experiment we found that participants made errors when trying to match the orientation of a moving line. In the second experiment we investigated how the matching errors in the matching task were related to the errors made in a visual matching task. In particular, we tested whether participants were using the last perceived orientation of the moving line to match its orientation or that some kind of averaged perceived orientation was used.

In addition to correctly orienting the hand, a correct timing of the movement is needed for a successful catch. The timing aspect of catching movements has been studied extensively in experiments in which participants had to catch a ball (for an overview, see Savelsbergh \& Whiting, 1996). In these experiments a ball was thrown towards the participant and at some time during the movement the ball was made invisible. Subjects were instructed to catch the ball, but they might fail to do so due to a lack of visual information. The number of times the ball was caught was measured as a function of the time during which the ball was visible ('the visible period') and the time period in which the ball was invisible ('the occluded period'). Both the visible and the occluded period were found to affect the percentage of balls caught (Sharp \& Whiting, 1974).

The so-called 'motion extrapolation' experiments provide additional information on the timing of catching movements. In motion extrapolation experiments a target approaching the participant becomes invisible at some point during its movement. The task of the participants is to estimate the point in time when the target arrives at a predefined target location. In general, participants could accurately predict the arrival time of the hidden object (Rosenbaum, 1975; Sokolov, Ehrenstein, Pavlova, \& Cavonius, 1997; Wiener, 1962). Only when the object was hidden for more than a second, performance started to deteriorate (Lyon \& Waag, 1995).

The movement data of the matching tasks in Experiments 1 and 2 show that participants made their interception movements too late. The hand tended to arrive at the interception point after the target passed through. By performing an additional analysis on the data of Experiments 1 and 2 we investigated whether differences in arrival time of the hand-held bar affected the accuracy of matching. In Experiment 3 we tested whether participants completed their arm movement to the screen after the line passed through the interception point because they could not estimate the arrival time of the hidden line correctly. Participants were asked to perform an extrapolation task. In this task they watched a line approaching them. Halfway the 
screen the line became invisible. The task of the participants was to press a button at the moment they thought the hidden line passed through a predefined location.

\section{Experiment 1}

In the first experiment we investigated how participants orient their hand when they have to catch an approaching line. Two catching situations were studied. In one situation the line was visible at all times throughout the movement to the participant while in the other situation it became invisible before arriving at the interception point. Participants were asked to match the orientation of the line when they thought it passed through the interception point by pressing a hand-held bar on the screen. By measuring the orientation of the bar the orientation of the hand could be determined when participants would try to "catch" the line.

\subsection{Method}

\subsubsection{Participants}

Eight participants took part in the experiment. Two of them were the authors. The others were naive with respect to the purpose of the experiment. These participants were paid for their participation. All participants had normal or corrected-tonormal vision. They were all right-handed.

\subsubsection{Apparatus}

An LCD projector (Philips 4750), connected to a PC, was used for the presentation of the stimuli. The stimuli were projected within a $142 \times 105 \mathrm{~cm}$ computer display image on a $2.5 \times 2 \mathrm{~m}$ vertical projection screen. Participants were asked to bring a $29 \mathrm{~cm}$ long bar to a predefined interception point on the screen in the same orientation as the moving line at the time that the line would pass through the interception point. The orientation of the bar was measured using an Optotrak 3020 system (Northern Digital Inc.), which measured the position of two infra-red light-emitting diodes (IREDs), attached to the bar at a distance of $20 \mathrm{~cm}$. The location of the IREDS was sampled at a frequency of $50 \mathrm{~Hz}$. The orientation of the bar could be measured with an accuracy better than $0.5 \mathrm{deg}$.

The set-up used in this experiment was the same as that in a previous experiment (Hermens \& Gielen, 2003), where subjects could see the edges of the projection screen. In that study, we demonstrated a clear oblique effect in the perception of slanted visual lines. Since the aim of this study was to investigate similarities in the perception and grasping of oriented lines, we did not attempt to hide the edges of the screen. The projection screen did not have any visible texture.

\subsubsection{Stimuli}

The stimuli consisted of solid lines presented on a vertical screen. The lines moved at a speed of $62 \mathrm{~cm} / \mathrm{s}$ along a horizontal path towards the participant starting their movement at a location $120 \mathrm{~cm}$ from the left side of the image display. A predefined 


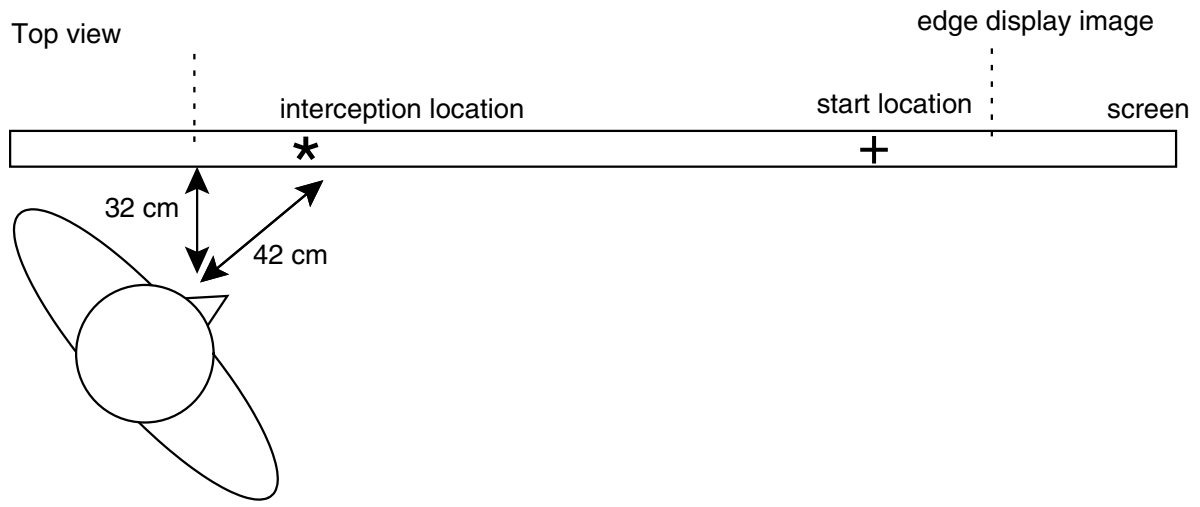

Fig. 1. The position of the participants with respect to the screen. Dotted lines show the location of the left and right side of the display image. '+' and '-' signs indicate the start location of the moving line and interception location respectively.

interception point was used which was kept constant across trials at $20 \mathrm{~cm}$ from the left side and $22 \mathrm{~cm}$ from the top of the computer display image. The starting position and the interception point location with respect to the participant is illustrated in Fig. 1 .

During its path the moving line (1) either moved across the visual display all the way from the right to the left or it disappeared (2) halfway the display image, $75 \mathrm{~cm}$ from the left, or (3) just before the location at which the orientation had to be matched, $27 \mathrm{~cm}$ from the left of the display image. In the condition in which the line disappeared outside the screen participants could see whether the orientation of the bar matched the orientation of the moving line when they tried to cover it at the interception point. This might have provided feedback on the accuracy of the selected orientations. By having the line disappear just before the interception point the role of on-line visual feedback on the matching performance could be investigated.

All lines were presented $22 \mathrm{~cm}$ below the top side of the display image. At this height, the lines moved about $10 \mathrm{~cm}$ above eye height of the subjects.

\subsubsection{Design}

The orientation of the moving lines could be horizontal ( $0 \mathrm{deg})$, vertical (90 deg) or oblique ( $+45 \mathrm{deg}$ or $-45 \mathrm{deg}$ ) with $+45 \mathrm{deg}$ defined to be a counter-clockwise rotation of the horizontal line by $45 \mathrm{deg}$. To stress the importance of matching the line's orientation and not a preconceived orientation, an additional scatter of 2, 4, or 6 deg was added to each of the main orientations. For example, for the $45 \mathrm{deg}$ orientation the orientations $39,41,43,47,49$, and $51 \mathrm{deg}$ were presented. The lines could disappear at three possible locations, as described in the stimuli section. The combination of four main orientations, six orientations near these main orientations, three disappearance locations, and two repeated presentations resulted in a total of 144 trials. Each mean per participant used in the statistical analysis of the data was 
therefore based on 12 repeated measurements. The order of trials was randomized across participants.

\subsubsection{Procedure}

Participants were standing at the left of the projection screen on which the image of a moving line was projected using the LCD projector. Subjects viewed the screen from an oblique angle with the cyclopean eye at a distance of $32 \mathrm{~cm}$ from the screen, just in front of the left edge of the computer display image. The distance from the cyclopean eye to the intersection point of the screen straight ahead was $42 \mathrm{~cm}$ (see Fig. 1).

At the start of each trial participants held the bar in their right hand near their waist. Before the line started to move, the line was shown at the starting position for $500 \mathrm{~ms}$. After the line started to move participants had to bring the bar to the interception point with the proper orientation at the proper time, irrespective of whether the line continued to be visible during its movement across the screen or whether it disappeared during it's movement to the interception point. After touching the screen with the bar the participants had to keep the bar at that location and orientation until a sound indicated the end of the trial $2 \mathrm{~s}$ after motion onset. Between the auditory signal and the beginning of the next trial there was a 2 s delay. The entire session took about $30 \mathrm{~min}$.

\subsubsection{Data analysis}

The orientation of the bar was determined by measuring the location of two IREDs mounted on the bar at a distance of $20 \mathrm{~cm}$. To obtain a good estimate of the matched orientation, the mean IRED location of the final 50 samples of each sample period were used. The orientation of the bar was computed by fitting a line through the mean locations of the two IREDs at the bar. Trials in which participants moved the bar during the final 50 samples (about one trial per participant), or in which one of the IREDs was not visible to the Optotrak system (about five trials per participant) were removed from the data analysis.

For each main orientation and each location at which the line disappeared a $t$-test was carried out to test whether the orientation of the hand-held bar was different from the orientation of the moving line. The significance level for these tests was corrected for the number of tests performed using a Bonferroni correction. An analysis of variance tested the interaction between line orientation and disappearance location. Paired samples $t$-tests were used to determine whether the location at which the line disappeared had an effect on the size of the signed differences between bar and line orientation. Two-tailed tests were used.

\subsection{Results and discussion}

Fig. 2 shows the matching orientations together with the actual orientations of the moving line for each of the disappearance locations. Data of all participants have been plotted in one figure, since the pattern of results was similar across participants. Near the reference lines symbols are inserted which indicate the results of the statis- 


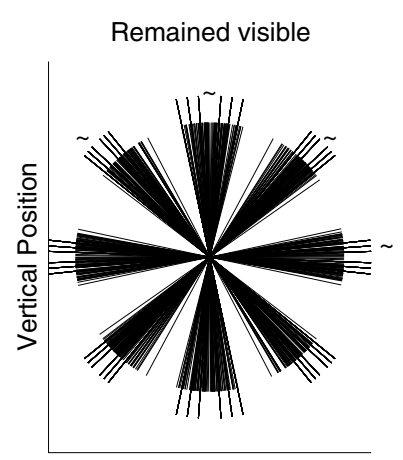

Horizontal Position

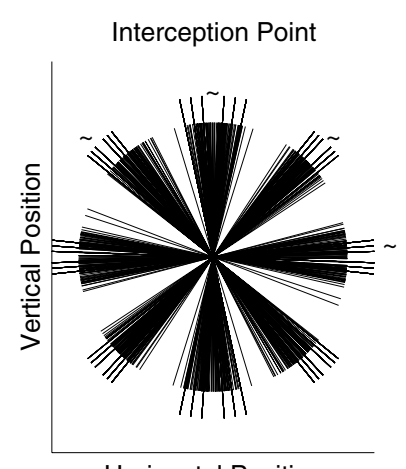

Horizontal Position

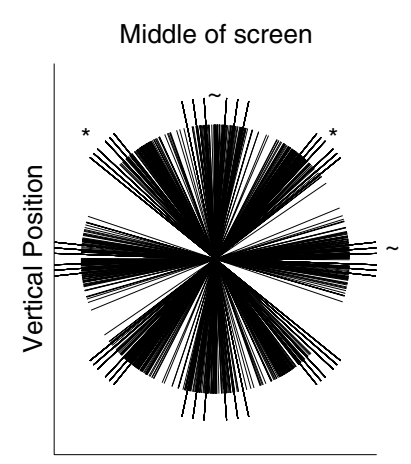

Horizontal Position

Fig. 2. Matching orientations (shorter lines) together with the reference orientations (longer lines) for the three disappearance positions of the moving line. Data from all eight participants are shown. A single asterisks denotes a significant difference between matching and reference line orientation.

Table 1

Mean signed errors and standard deviations across participants observed in Experiment 1 for the three disappearance locations of the moving line

\begin{tabular}{lllcc}
\hline Disappearance & $0 \mathrm{deg}$ & $90 \mathrm{deg}$ & $45 \mathrm{deg}$ & \multicolumn{1}{c}{$-45 \mathrm{deg}$} \\
\hline Middle & $-1.4120(2.5751)$ & $2.9792(3.2205)$ & $-6.7783^{*}(2.7048)$ & $11.6316^{*}(3.4823)$ \\
Interception point & $-0.4514(1.3967)$ & $1.8789(2.0533)$ & $0.3411(2.0375)$ & $5.0916(2.4741)$ \\
Outside screen & $-1.0322(3.3315)$ & $1.2180(2.0437)$ & $-0.8263(1.2998)$ & $4.3302(2.4787)$ \\
\hline
\end{tabular}

An asterisk denotes a significant deviation of the mean from zero.

tical test to compare matching and reference orientations. Mean errors and standard deviations across participants are shown in Table 1.

In an analysis of variance the effects of disappearance location and line orientatation on signed matching errors were tested. A significant interaction effect between disappearance location and line orientation was found $(F(2,6)=71.223, p=0.014)$.

For the disappearance location halfway the screen large systematic errors were found for the oblique orientations. The orientation of lines disappearing later was matched more accurately. The signed errors for oblique orientations for the disappearance location halfway the screen differed significantly from that for the other conditions (all $p$-values smaller than 0.001 ). For the orthogonal orientations no effect of disappearance location was found.

For all three conditions the size of the errors for the -45 deg orientation was larger than that for the $45 \mathrm{deg}$ orientation (all $p$-values smaller than 0.035).

An additional analysis of variance tested the effects of disappearance location and line orientation on the unsigned matching errors. No significant interaction effect of disappearance location or line orientation on unsigned errors was found $(p>0.3)$. The two main effects of disappearance location and line orientation were significant (both $p$-values smaller than 0.01). Paired comparisons of unsigned errors of different line orientations showed that the unsigned errors of the vertical line orientation were different from those of the other line orientations (all $p$-values smaller than 0.01). 
These effects differ from those found by Hermens and Gielen (2003). In their study the two orthogonal orientations showed larger unsigned errors, while in the catching situation no different unsigned errors were found for horizontal lines and oblique lines. Paired comparisons of the unsigned errors for the different disappearance locations showed that these errors were larger for lines becoming invisible halfway the screen, compared to those becoming invisible later on during the movement (both $p$-values smaller than 0.01). The difference between the unsigned errors for early and late disappearing lines could reflect an effect of visual feedback.

A possible source of the observed signed errors might be the incorrect visual perception of line orientation. Hermens and Gielen (2003) have shown that participants incorrectly match the orientation of visually presented oblique lines. If errors in visual perception were underlying the matching errors of moving lines, this might suggest that the action system is susceptible to perceptual errors. However, there might be a distinction in the kind of errors that the action system is susceptible to. All illusions used to demonstrate the existence of separate pathways for action and perception made use of information of one object relative to another object. In the Müller-Lyer illusion, for example, the size of the bar is perceived incorrectly because of the wings in the illusion. The perceptual errors in orientation perception are independent of any objects in the neighborhood of the object of which the orientation has to be estimated. Therefore, the action system might be susceptible to orientation perception errors, but not to size estimation errors.

To investigate whether there is a relation between the matching errors of Experiment 1 and visual matching errors, the size of the visual matching errors was measured in Experiment 2 to allow for a comparison. Therefore, the reference line location was varied systematically in one of the visual matching tasks of Experiment 2.

In Experiment 2 we also investigated whether orientation of the hand-held bar in Experiment 1 could be related to the orientation of the moving line at some position on its trajectory to the participant. Specifically, we investigated whether participants used an average across a fixed sampling period to match the orientation of the moving line. A possible averaging of perceived orientation might be carried out for the last perceived orientations before the disappearance of the moving line. However, another alternative might be that subjects track the moving line at the beginning of the movement and then make a saccadic eye movement to the interception point (c.f., Johansson, Westling, Backstrom, \& Flanagan, 2001; Neggers \& Bekkering, 2000, 2001). According to this strategy, any errors might be related to errors in the percept of line orientation near the starting location of the moving line. In order to test whether participants use this strategy, we have varied the starting location of the moving line, and we expect that matching errors will be larger for lines which start their movement farther away from the participant.

\section{Experiment 2}

In the first experiment we found that participants made errors when they had to match the orientation of a moving line. In the second experiment we will further ex- 
plore various hypotheses regarding the origin and nature of these errors. For this purpose, the participants were asked to carry out four different matching tasks.

Participants were tested in two visual matching tasks to compare the errors in Experiment 1 with errors in the visual perception of the orientation of a static line at different positions relative to the matching line. In addition, two additional matching tasks with moving lines were used to investigate possible strategies that participants might have used to match the orientation of the moving line in Experiment 1.

In the visual matching tasks the participants had to rotate a line ('the matching line') until it was perceived to be parallel to a reference line. The first visual matching task involved matching orientations of orthogonal ( 0 and $90 \mathrm{deg}$ ) and oblique orientations ( 45 and $-45 \mathrm{deg}$ ). The reference line was either at the location where the line started its movement in Experiment 1 or at the location halfway the image display, where the moving line disappeared. The matching line was always at the location of the interception point of Experiment 1. If the matching errors found in Experiment 1 reflect errors in the visual perception of line orientation, the same pattern of constant and variable errors is expected as in Experiment 1.

In the second visual matching task, the participants had to match the orientation of the oblique reference lines only. To study the effect of the position of the reference line on the orientation of the matching line, the position of the reference line was varied systematically relative to the position of the matching line. The matching line was always at the interception point of Experiment 1, while the reference line was at various horizontal distances with respect to the matching line.

The third matching task was similar to that in the first experiment with the only difference that the line could move at two different velocities. The lines always became invisible halfway the screen. The speed of the line was varied to investigate whether participants might use an averaged perceived line orientation across a fixed period to match the orientation of the moving line. Since a faster moving line moves over a longer spatial interval within a fixed time period, averaging of perceived line orientations in a fixed time interval implies that perceived orientations of more distant lines are taken into account in the estimation of line orientation for higher movement velocities. If participants used a fixed time interval for averaging perceived orientation, faster moving lines are expected to result in larger matching errors.

In the fourth matching task the moving reference line, of which the orientation had to be matched, started its movement either at a short or a long distance from the participants. Earlier experiments by Johansson et al. (2001) demonstrated that participants track a moving object with their eyes for a short time. In their experiment participants were asked to move an object to a predefined target location. Early after movement onset participants moved their eyes from the moving object to the target location. By varying the distance at which the line started its movement we investigated whether participants looked away from the moving line early after movement onset. If participants of Experiment 1 looked away from the moving line early after the line's movement onset, errors are expected to be larger for lines starting their movement at a long distance than for lines starting at a short distance. 


\subsection{Method}

\subsubsection{Participants}

The number of participants in the four matching tasks was $6,6,7$, and 6 , respectively. The two authors (FH and SG) participated in all four matching tasks. The other participants were naive with respect to the purpose of the experiment. The participants, who were not members of the department of Biophysics, were paid for taking part. All participants had normal or corrected-to-normal vision.

\subsubsection{Apparatus}

For stimulus presentation the same PC and LCD projector were used as in Experiment 1. In the visual matching tasks the orientation of one of the projected lines ('the matching line') could be adjusted using a computer keyboard. The equipment used for matching the orientation of the moving lines was identical to that used in Experiment 1 .

\subsubsection{Stimuli}

In all matching tasks computer-generated lines served as stimuli. The lines used in the visual matching tasks consisted of seven dots each at $13 \mathrm{~mm}$ distance within the $142 \times 105 \mathrm{~cm}$ display image, plotted in white on a black background. Because participants could spend as much time as they wanted in the visual matching task, seven dots were used instead of a solid line. A solid line would have allowed participants to estimate its orientation by looking at the staircase pattern within the line, which originated from the finite resolution of the visual display in graphics mode.

The group of participants performing the first of the two visual matching tasks were presented with a reference line either in the upper right part of the screen with the center $122 \mathrm{~cm}$ from the left side of the display image, or at the middle of the screen $67 \mathrm{~cm}$ from the left side. The matching line was always presented in the upper left part of the screen with its center at the interception point in Experiment 1, which was at the same height as the reference line, at a distance of $20 \mathrm{~cm}$ from the left side of the display image.

In the second visual matching task the reference line could appear at one of eight possible locations, namely at $44,55,67,78,89,100,111$, or $122 \mathrm{~cm}$ from the left of the display image. The orientation of this reference line had to be matched by rotating the matching line, which was always presented at the upper left part of the screen at the interception point in Experiment 1 with its center $20 \mathrm{~cm}$ from the left side.

In the third matching task the paradigm was the same as that in Experiment 1. The only difference was that the line could move at a low speed $(31 \mathrm{~cm} / \mathrm{s})$ or at a high speed $(62 \mathrm{~cm} / \mathrm{s}$, which was the same as that in Experiment 1). The line started its movement $122 \mathrm{~cm}$ from the left side of the display image and it always disappeared halfway the computer display ( $67 \mathrm{~cm}$ from the left side of the display image).

In the fourth matching task the moving lines were visible in two different parts of the visual display. Half of the lines started their movement $106 \mathrm{~cm}$ from left side of the display image, while the other half started at $75 \mathrm{~cm}$ from the left. All lines disappeared when their center was at $44 \mathrm{~cm}$ from the left of the display image. 
The lines in the four matching tasks were all presented at the same height as in Experiment 1, about $10 \mathrm{~cm}$ above eye height.

\subsubsection{Design}

For the first visual matching task four main orientations of the reference line (45, $-45,0$, and $90 \mathrm{deg}$ with respect to the horizontal) were used. In the other tasks only the two oblique orientations ( 45 and $-45 \mathrm{deg}$ ) were used.

For the visual matching tasks (the first two tasks) an additional scatter of 2 or 4 deg was added to the four main orientations. For example, for the $45 \mathrm{deg}$ orientation, the reference line could be presented with equal probability at an orientation of 41, 43, 45, 47, or $49 \mathrm{deg}$. For the matching tasks with moving lines the main orientation itself was not presented. Instead orientations plus or minus 2, 4, or $6 \mathrm{deg}$ relative to the main orientation were used. The participants were told that some scatter had been added to the orientation of the line, so that it would be important to align the orientation of the matching line with that of the reference line, instead of, for example, matching some preconceived orientation relative to external cues, such as gravity or the edges of the screen.

For the visual matching tasks each combination of main orientation and reference line location was presented ten times to each participant. In the other two matching tasks, each condition was tested 12 times. The order of the trials was randomized for each participant.

\subsubsection{Procedure}

The participants performing the visual matching task were seated in a chair at the left side of the projection screen at the same location where participants were standing in Experiment 1. The height of the chair was adjusted such that the participants were viewing the screen at about the same height as they would be viewing it when they were standing.

At the beginning of each visual matching trial two lines appeared on the screen, each consisting of seven dots, and the participant was asked to rotate the matching line using the computer keyboard such that its orientation matched that of the reference line. The orientation of the matching line could be adjusted in steps of $2,0.5$, or $0.1 \mathrm{deg}$. The participant pressed a button when satisfied with the selected orientation. After the button was pressed the screen was cleared and after a $1000 \mathrm{~ms}$ intertrial interval two new lines appeared on the screen.

Participants matching the orientations of moving lines carried out the same task as the participants of Experiment 1. They pressed a hand-held bar on the predefined interception point on the screen at the moment they thought the invisible line would pass through the interception point. They tried to hold the bar in the same orientation as the moving line.

\subsubsection{Data analysis}

To test for systematic errors in the visual matching tasks a signed error was computed as the difference between the orientation of the reference line and that of the matching line. The signed error for the matching task with moving lines was 
computed by taking the difference between the orientation of the hand-held bar and the orientation of the moving line. Statistical tests using means per participant were used to test whether the signed errors were different from zero. For the first visual matching tasks, where eight paired $t$-tests were carried out, a Bonferroni correction was applied to the significance level.

\subsection{Results}

Fig. 3 shows the orientations of the reference lines (longer lines) and corresponding matching lines (shorter lines) for the first visual matching task. The orientations at the left and right side of Fig. 3 correspond to the matching orientations for reference lines at the middle ( $67 \mathrm{~cm}$ to the left of the edge of the computer display image) and upper right side (122 cm from the edge of the computer display image) of the computer visual display, respectively. Since similar results were obtained for each of the participants, data from all participants were plotted in one figure. Tilde and asterisk symbols in the plot show the outcomes of statistical tests using means per participants. A tilde-sign denotes a non-significant difference between the reference and matching orientations. A single asterisk refers to a difference at a significance level of 0.05 .

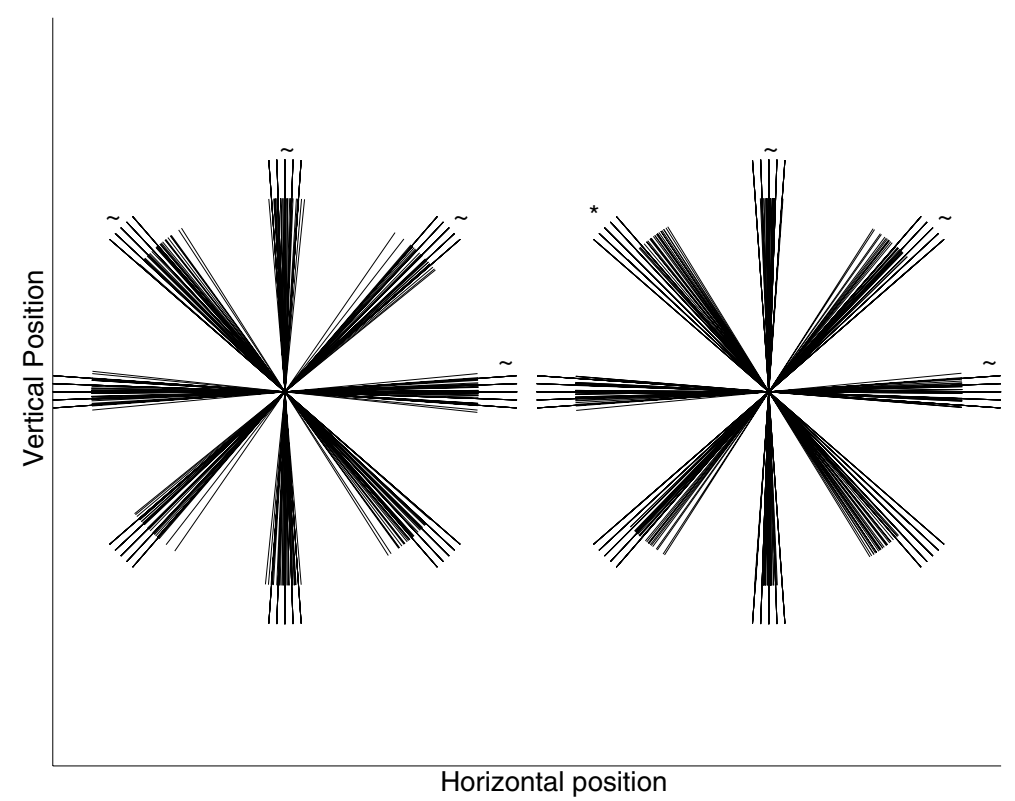

Fig. 3. Matching line orientations (shorter lines) together with the reference line orientations (longer lines) for the two main reference line locations. The left reference lines were presented at a distance of $67 \mathrm{~cm}$ from the left side of the display image. The right reference lines were presented $122 \mathrm{~cm}$ from the left side. A tilde symbol near the lines denotes a non-significant difference between matching and reference line orientations. A single asterisk denotes a significant difference at a significance level of 0.05 . 
Table 2

Mean signed errors and standard deviations across participants found for the visual matching task with two main reference line locations

\begin{tabular}{lrrrl}
\hline Reference location & $0 \mathrm{deg}$ & $90 \mathrm{deg}$ & $45 \mathrm{deg}$ & $-45 \mathrm{deg}$ \\
\hline Middle of the screen & $0.1250(0.3781)$ & $0.1767(1.0320)$ & $-0.6650(2.5447)$ & $1.1000(5.3240)$ \\
Right of the screen & $-0.1083(0.5899)$ & $-0.2380(1.2825)$ & $-4.7117(4.0870)$ & $7.1161^{*}(2.7429)$ \\
\hline
\end{tabular}

Asterisks denote a significant difference between matching and reference line orientation.

Mean signed errors and their standard deviations for the first visual matching task are listed in Table 2. An analysis of variance tested the effects of reference line location and line orientation on signed errors. A significant interaction effect between reference line location and orientation was found $(p<0.05)$.

As in Experiment 1, small mean errors were found for the 0 and 90 deg orientations and larger errors were observed for the -45 and 45 deg orientations. Only for -45 deg orientations at the upper right position significant errors were found.

The errors for the oblique orientations in Experiment 1 and the visual matching task were compared using a repeated measures ANOVA. Data of participants who took part in only one of the two matching tasks were used in the test. The errors for the middle disappearance location of Experiment 1 and those for the upper right matching location of the visual matching task were compared. A significant interaction effect was found between the orientation of the reference line $(+45 \mathrm{deg}$ or -45 deg) and the matching task ("catching" vs visual matching). Tests of simple effects showed that there was no effect of matching task for the $-45 \mathrm{deg}$ orientation $(p=0.224)$, while the errors for the $+45 \mathrm{deg}$ orientation were significantly different for the two matching tasks $(p=0.001)$.

A paired samples $t$-test demonstrated that the effect of reference location was significant for the two oblique orientations (both $p$-values smaller than 0.006). Larger errors were found for more distant reference line locations. This result suggests that errors increase with increasing distance between the matching and the reference line.

In the second visual matching task the influence of the distance between the two lines was investigated in more detail. This was done by systematically varying the horizontal position of the reference line while keeping the matching line at the same position.

The results of the second visual matching task are shown in Fig. 4. In this figure unsigned mean differences between the orientation of the matching and the reference line are shown for each participant. The means across participants are shown in the last subplot of the figure. Unsigned errors are shown to allow for a comparison of the size of the errors for the two types of oblique lines $(-45$ and $+45 \mathrm{deg})$. If participants performed the matching task solely on the basis of the orientation of the retinal image of the lines the size of the errors was expected to be equal for both types of oblique lines. A $t$-test using means per distance to the reference line determined the significance of the mean error difference for the -45 and the $+45 \mathrm{deg}$ orientation. For three of the seven participants the difference in the size of the errors for the -45 and the +45 deg orientation was significant $(p<0.005)$. The plots of Fig. 4 show that errors increase with the distance of the reference line towards the observer. 

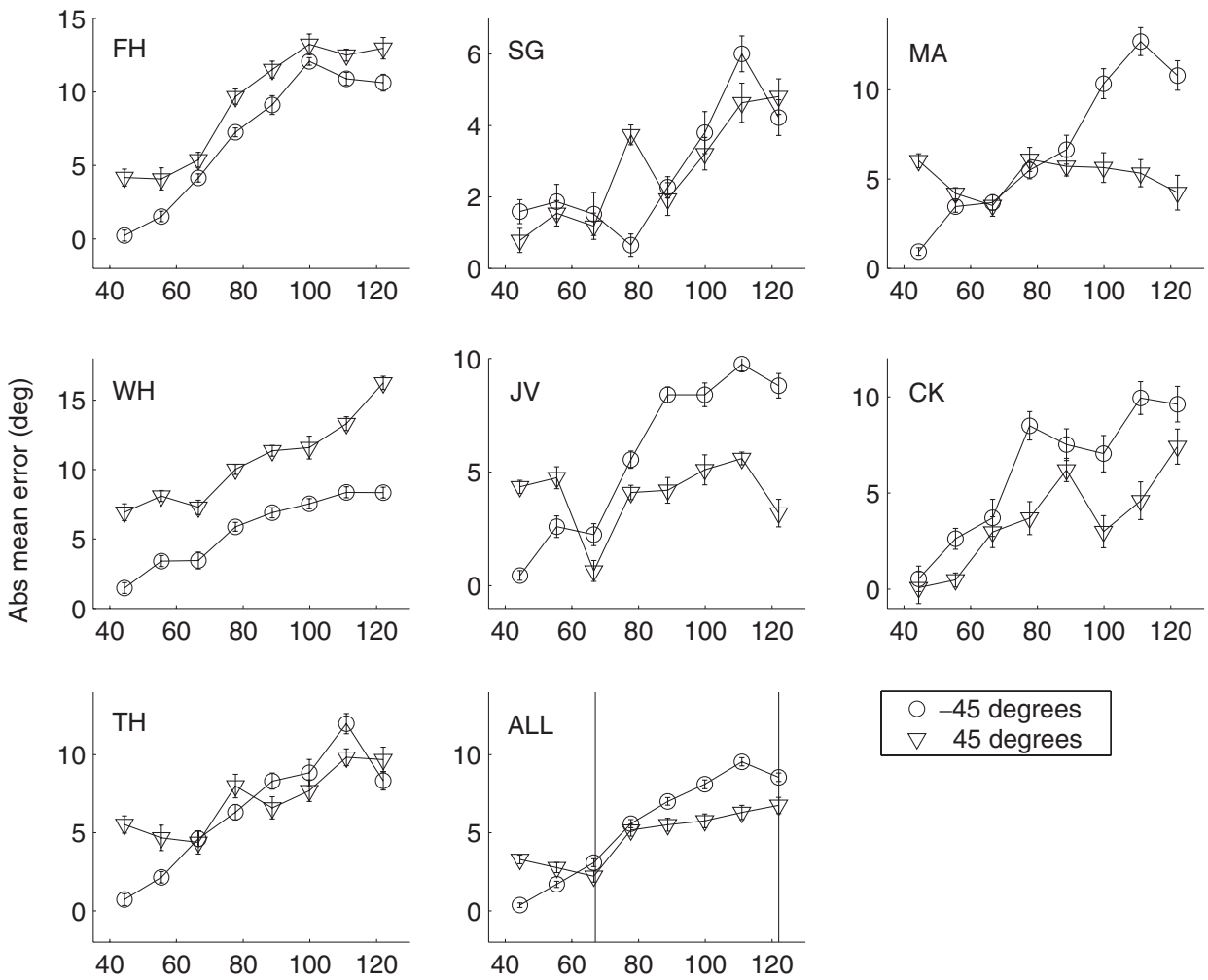

Distance to the reference line from the left of the display image $(\mathrm{cm})$

Fig. 4. Unsigned mean differences between matching line and reference line orientation plotted against the distance of the reference line towards the left side of the display image. In the last subplot the means across participants are shown. The vertical lines show the distances at which the reference lines in the first visual matching task were presented.

In Experiment 1 the participants made significant errors in matching for the reference line, which disappeared at a distance of $75 \mathrm{~cm}$ from the left side of the display image. A comparison of the data in Figs. 1 and 4 shows that the errors for the condition, in which the reference line disappeared halfway the screen in Experiment 1, are considerably larger than that at the corresponding distance in Fig. 4. The mean error for the "catching" task was near $8 \mathrm{deg}$, while the mean visual matching error for a reference line at a distance of $75 \mathrm{~cm}$ was close to $3 \mathrm{deg}$. This means that participants of Experiment 1 did not use the final perceived orientation of the moving line to match its orientation.

In two subsequent matching experiments we tested two other possible strategies which subjects of Experiment 1 could have used. First a strategy in which participants based their matching orientations on some time-averaged perceived orientation of the moving line. If participants used such a time-averaged perceived orientation the speed of the moving line was expected to have an effect on matching 
accuracy. Fig. 5 shows the mean errors for each participant for the two line speeds. Mean signed errors and standard deviations are shown in Table 3. In this table the results of statistical tests are included. These tests determined the significance of the difference between the actual orientation of the moving line and the orientation of the bar held by the participants.

An analysis of variance with line speed and line orientation as factors demonstrated an effect of line orientation $(F(1,5)=30.4, p=0.03)$, but no significant interaction effect between line speed and line orientation. Also no significant main effect of line orientation was found $(p>0.4)$.

The second matching task with moving lines investigated whether participants estimate the orientation of the moving line from the first part of the movement. A group of participants matched the orientation of moving lines starting at two different starting positions. Mean errors for each participant and for each starting position of the moving line are shown in Fig. 6. The mean errors and their standard deviations are listed in Table 4 . Within the $-45 \mathrm{deg}$ orientation the mean error was larger for almost each participant when the line started its movement at a more distant position. An analysis of variance tested the effects of starting location and line orientation and their interaction. The interaction effect was marginally significant
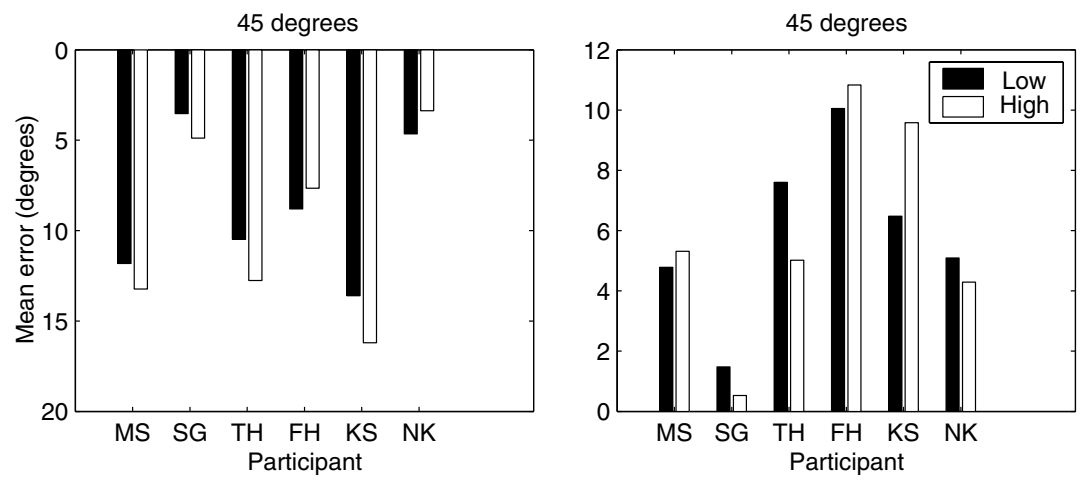

Fig. 5. Mean difference between the orientation of the moving line and the hand-held bar. The mean differences for the moving lines with an orientation of $-45 \mathrm{deg}$ are shown in the left panel, and those for the lines with an orientation $+45 \mathrm{deg}$ are shown in the right panel. The black bars show the mean difference for the slowly moving lines, and the white bars for the bars moving at a high speed.

Table 3

Mean signed errors and standard deviations across participants for the matching task with lines moving at one of two possible speeds. In the plot a tilde-sign shows that the signed errors were not significantly different from zero

\begin{tabular}{lll}
\hline Speed & $45 \mathrm{deg}$ & $-45 \mathrm{deg}$ \\
\hline Low & $-8.8117^{*}(3.9986)$ & $5.9133^{*}(2.8946)$ \\
High & $-9.6833^{*}(5.1280)$ & $5.9283^{*}(3.7548)$ \\
\hline
\end{tabular}

An asterisk represents a significant difference at a significance level of 0.05 . 

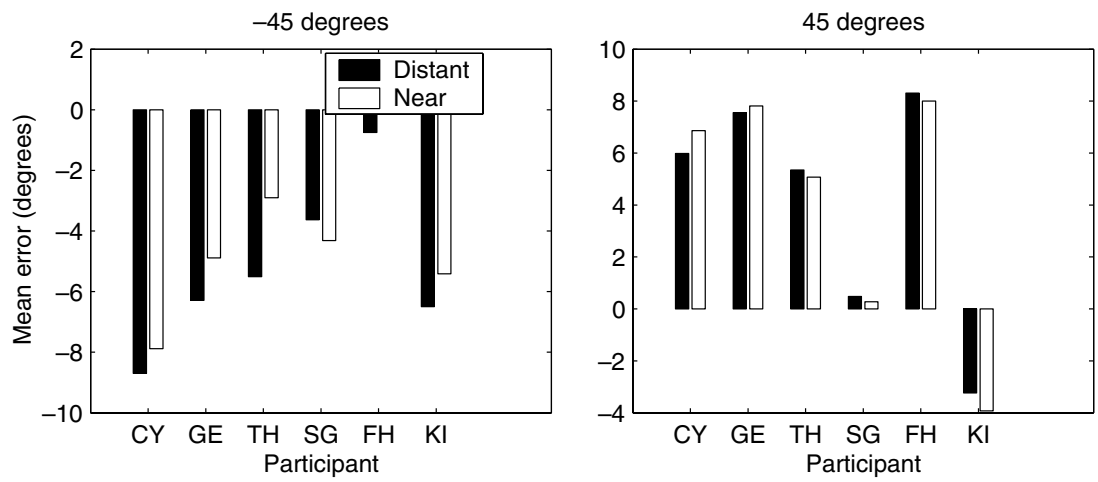

Fig. 6. Mean errors for each participant in the matching task in which lines started moving at one of two possible locations. The black bars show the means for lines starting their movement at a distant location, and white bars for lines starting at a near by location. The left panel shows the errors for the -45 deg orientation, and the right panel the errors for the $+45 \mathrm{deg}$ orientation.

Table 4

Mean signed errors and standard deviations across participants for the matching task in which the moving line started its movements at one of two possible starting locations

\begin{tabular}{lll}
\hline Visible distance & $-45 \mathrm{deg}$ & $45 \mathrm{deg}$ \\
\hline Distant location & $-5.2300^{*}(2.7381)$ & $4.071(4.5100)$ \\
Near location & $-4.1517^{*}(2.7997)$ & $4.0150(4.8266)$ \\
\hline
\end{tabular}

An asterisk represents a significant difference at a significance level of 0.05 .

$(p=0.074)$. A $t$-test showed that within the $-45 \mathrm{deg}$ orientation the mean error was larger for lines starting their movement at a more distant location $(t(5)=-2.488$, $p=0.028)$. No significant effect of starting location was found for the $45 \mathrm{deg}$ line orientation.

\subsection{Discussion}

In the first visual matching task, in which participants matched both orthogonal and oblique orientations, a qualitatively similar pattern of results was obtained as in the matching task of Experiment 1. No systematic errors were found for orthogonal line orientations both for the catching and the visual matching task, while for the two oblique orientations systematic effects were found in both tasks. In the matching task the orientation of the hand-held bar was tilted further towards the vertical when the line disappeared at a more distant location. In the visual matching task the orientation of the matching line was set to a more vertical orientation when the reference line was presented at a larger distance. The qualitative similarity of the outcomes of the visual matching task with static lines and the haptic matching task with moving lines suggest that participants selected the orientation of the hand-held bar on the basis of the perceived orientation of the moving line. 
When reference line orientation was varied systematically, a sigmoidal relationship was found between perceived orientation and distance towards the line. The function of perceived error appears to be sigmoidal with the steepest increase in error at a distance of about $80 \mathrm{~cm}$ from the left of the display image.

It is well known that geometric distortion causes deviations between line orientation in space and orientation of the line on the retina. In order to investigate the effect of geometric distortion on visual matching errors, we calculated the expected retinal image of two lines presented on a frontal plane to see whether participants perform the matching task using the orientations in the retinal image only. For the computations the lines were assumed to be presented at eye height. The distance from the eye lens to the retina was set to $1 \mathrm{~cm}$, although any other value could have been chosen (e.g., Pizlo, 1994). The predicted error as a function of the distance of the reference line is shown in Fig. 7. The function intersects with the horizontal axis for a reference lines at $20 \mathrm{~cm}$, which it should, since the matching line was presented at this distance. What is not visible within the range of 0 to $122 \mathrm{~cm}$ is that the function asymptotically approaches an error size of 45 deg for larger distances. The 45 deg error is approached because the lines were presented at an orientation of 45 deg and the distortion causes the perceived line orientation to approach $90 \mathrm{deg}$.

Some participants showed larger errors than those predicted on the basis of perspective, but on average the size of the errors of the prediction and of the

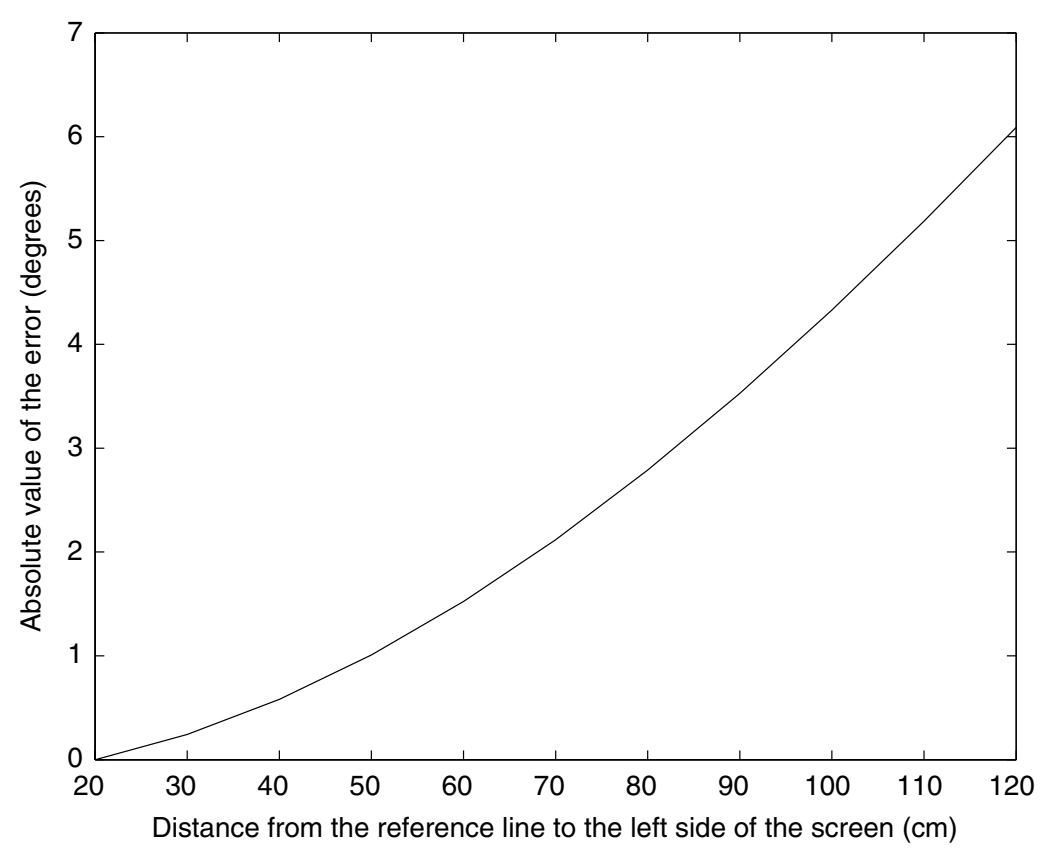

Fig. 7. Predicted absolute mean errors for the -45 and the 45 deg orientation, if participants use the orientations on the retinal image to match the orientations of the line. 
observations correspond quite accurately. One might argue that the matching tasks in Experiments 1 and 2 were ambiguous, since participants might have thought that the lines on the retina should be matched in orientation. However, some participants who took part in two experimental tasks were presented with the systematic errors they made in the first of the two tasks. No differences were found between the errors of participants who were new to the task and participants who knew about the systematic errors they made in the previous task. This suggests that participants could not correct for the retinal distortion even when they knew that such a distortion occurred.

The matching task in which the speed of the moving lines was varied demonstrated that participants did not use a fixed time interval to average the perceived orientation of the reference line. Possibly, a small effect of line speed was present in the data, which might not have become obvious because of a lack of statistical power. Still, if an effect of line speed was actually present in the data, its direction was not consistent across participants.

Instead, it is more likely that participants looked away from the moving line early after its movement onset as demonstrated by the significant effect of the starting position of the moving line. Such a viewing strategy has been shown to occur in an experiment by Johansson et al. (2001). Participants, who were instructed to move a bar to a target location, did not follow the hand with their eyes all the way to the target location. Instead they tracked the object for a short time and then made a saccade to the target location early after movement onset. The results of our study suggest that participants follow a similar strategy as that reported by Johansson et al. (2001). The orientation errors were related to the errors in the perceived orientation early after movement onset.

\section{Experiment 3}

Participants of Experiments 1 and 2 were asked to press the hand-held bar at the screen at the very moment they thought the hidden line passed through the interception point. The moment at which the movement of the bar to the screen ended could be estimated from the movement data of Experiments 1 and 2. In Fig. 8 the mean time between the disappearance of the line and the end of the interception movement is plotted. The left panel of Fig. 8 shows the timing data of the fourth matching task of Experiment 2. In this matching task the lines were invisible across a distance of 24 $\mathrm{cm}$. In half of the trials the lines were visible across a long distance, and in the other half of the trials across a short distance. In the right panel the timing data of Experiment 1 are shown. Only the data of the lines invisible across a distance of $55 \mathrm{~cm}$ are shown, because the sampling periods (which started after the disappearance of the line) of the other two conditions were too short to estimate the end of the matching movement accurately. The horizontal lines in Fig. 8 indicate when the line actually reached the interception point. Since the lines in both experiments moved at the same speed, the arrival of the hidden line depends on the hidden distance only. Fig. 8 dem- 

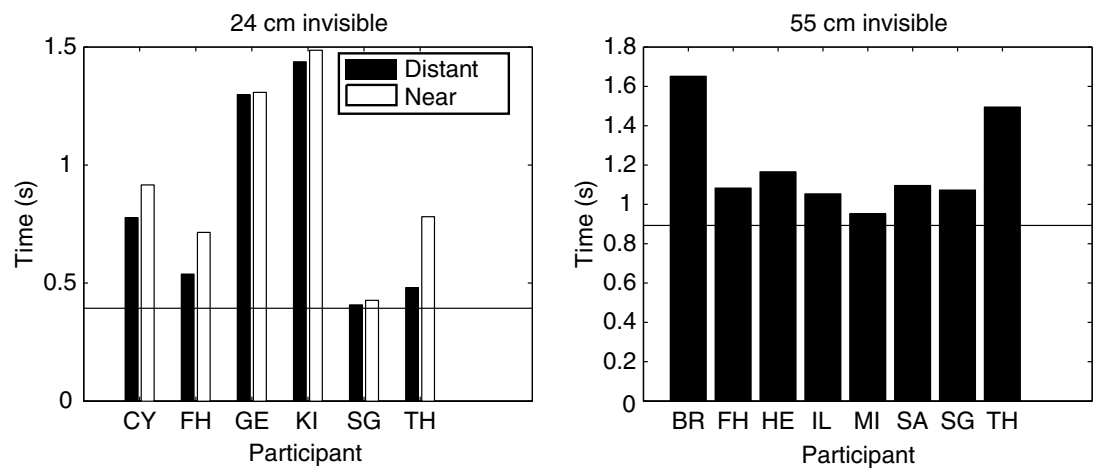

Fig. 8. Mean duration of the interval between the disappearance of the line and the end of the matching movement for each of the participants carrying out the fourth matching task of Experiment 2 (left panel) and of Experiment 1 (right panel). In the left panel the white bars show the time when the line started its movement at the near starting position. The black bars show the times for the lines starting at the distant starting position.

onstrates that in general participants ended their movement after the line had passed the interception point.

The important issue at stake is whether the errors in the timing of the movement affected the accuracy with which the orientation of the line was matched. In experiments in which participants were asked to grasp an object within a visual illusion, the effect of the illusion was found to increase with the delay between stimulus presentation and the onset of the grasping movement (Westwood et al., 2001). To investigate whether there was a relationship between the timing error and the orientation matching error, the correlation between the two errors was computed for each participant of Experiment 1 and of the fourth matching task in Experiment 2. Across participants the correlations for both matching tasks were close to zero. For Experiment 1 the mean correlation was -0.0839 and for Experiment 2 the mean correlation was -0.0471 , implying there was no relationship between timing errors and orientation matching errors. A previous study showed that the duration of the delay did affect the effect of an illusion on grasping (Westwood et al., 2001). We did not find a correlation between the duration until the end of the movement and the orientation error. This is in favor of the hypothesis that representations for action and perception of line orientation are the same.

Participants could have ended their interception movements too late because they overestimated the time the line needed to arrive at the interception point. A motion extrapolation experiment was carried out to investigate whether an overestimation of the hidden interval of the line caused the late interception movements. In this motion extrapolation experiment participants were presented with the same moving lines as in the first experiment. Their task was to press a button at the moment they thought the hidden line passed through a predefined interception point. If participants would overestimate the hidden interval, they would press the button too late. 


\subsection{Method}

\subsubsection{Participants}

Six participants took part in the experiment. Two of them were the authors. The other participants (students of the university of Nijmegen) were paid for their participation.

\subsubsection{Apparatus}

The same equipment was used as in Experiment 1. A button box connected to the parallel port of the computer was used to measure response times. The button box allowed response times to be measured with an accuracy better than $1 \mathrm{~ms}$.

\subsubsection{Stimuli}

The same lines as in Experiment 1 were used. They moved at a speed of either 31 or $62 \mathrm{~cm} / \mathrm{s}$. Each line started to move at one of three starting positions, namely 129 , 124 , or $120 \mathrm{~cm}$ from the left side of the display image. The lines became invisible either 71,77 , or $82 \mathrm{~cm}$ from the left of the display image. The starting and disappearance locations were varied to make sure that people would not base their responses on estimates of the interval from the starting position to the interception point or the interval from the disappearance location to the interception point. Lines were oriented at 0, 90, 45 and $-45 \mathrm{deg}$ with respect to the horizontal, as in Experiment 1. No scatter was added to each main orientation.

\subsubsection{Design}

The combination of two speeds, three starting positions, three disappearance positions, and four orientations resulted in a total of 72 trials. Each combination was presented twice resulting in a total of 144 trials. Participants needed about 25 min to complete the experiment.

\subsubsection{Procedure}

At the start of each trial the line appeared at its starting position where it stood still for $500 \mathrm{~ms}$. Then it started moving towards the participant either at a slow or a fast speed. When the center of the line arrived at the disappearance position, it became invisible. Participants were asked to press the button on the button box when they thought the line (which was invisible at that moment) passed through the predefined interception point. After they had pressed the button, they received feedback by a cross sign presented at the actual location of the line at the moment the button was pressed. The feedback was presented for $1000 \mathrm{~ms}$, followed by an intertrial interval of $2 \mathrm{~s}$. Each participant received 10 practice trials to get familiar with the task.

\subsection{Results}

First the data were inspected for practice effects. If performance would improve during the experiment, negative correlations were expected between trial number and signed error (the distance between the target location and the location of the line 
at button press). A mean correlation across participants between trial number and signed error of -0.0917 indicated that performance did not improve considerably during the course of the experiment. The very low correlation also means that participants did not use the feedback to improve the performance during the experiment.

In addition, autocorrelations of signed and unsigned errors were computed to check for intertrial effects. Maybe participants would try harder to correctly predict the correct location of the line after a trial with a large error. This would then result in a high negative autocorrelation for unsigned errors. If participants would use the strategy to press the button later on a trial after a trial in which they pressed too early and vice versa, a positive autocorrelation was expected for the signed errors. The average autocorrelation for unsigned errors was equal to 0.1417 , and that for the signed errors equal to -0.0617 . The low correlation values show that there were no intertrial effects.

A repeated measure ANOVA tested the effects of speed and orientation on the extrapolation accuracy. A significant effect of line speed was found $(F(1,5)=8.070$, $p=0.036)$. Line orientation did not have an effect $(F(3,3)=0.145, p=0.926)$. The interaction between speed and orientation was not significant $(F(3,3)=$ $0.683, p=0.619)$.

In Table 5 mean signed errors for each of the movement speeds and line orientations are shown.

\subsection{Discussion}

Participants could reasonably well estimate the moment at which the line passed through the interception point. For the slowly moving lines participants pressed the button too early, underestimating the duration of the hidden interval. This means that participants of Experiments 1 and 2 did not end their movements too late because they could not estimate when the hidden line arrived at the interception point. Instead, they might have focussed on matching the orientation of the hidden line which decreased the accuracy of the timing of the movements.

No effect of orientation of the line was found on extrapolation accuracy. This means that participants could correctly extrapolate the movement of the center of the line, ignoring the remainder of the line. Earlier Castet, Lorenceau, Shiffrar, and Bonnet (1993) found an effect of line orientation on speed estimates. We did not find an effect of line orientation on extrapolation accuracy. The long interval across which our lines were visible, might explain the difference in the effects of line orientation in the two experiments (Castet et al., 1993).

Table 5

Mean signed errors in $\mathrm{cm}$ (computed as the difference between actual location at button press and target location) and their standard deviations

\begin{tabular}{lllll}
\hline Speed & \multicolumn{1}{l}{0 deg } & $90 \mathrm{deg}$ & $45 \mathrm{deg}$ & \multicolumn{1}{c}{$-45 \mathrm{deg}$} \\
\hline Slow & $4.1886(3.2563)$ & $4.1210(3.3336)$ & $6.2062(4.9190)$ & $3.5559(2.7449)$ \\
Fast & $-0.7152(2.9619)$ & $0.7170(3.9526)$ & $-0.5447(3.1830)$ & $0.02699(3.7391)$ \\
\hline
\end{tabular}

Positive errors represent too early button presses. 
A significant effect of line speed on extrapolation accuracy was found. Participants mainly underestimated the time that the line needed to reach the target location when the line was moving at a low speed. Earlier Lyon and Waag (1995) showed that extrapolation accuracy decreased with the invisible period when lines were invisible for more than a second. In our experiment the slowly moving line was invisible for more than a second, which might explain the effect of line speed that we found.

\section{Conclusions}

When participants had to match the orientation of an approaching line they made systematic errors. The errors became larger when the line disappeared farther away from the participant. Qualitatively the errors matched those made in a visual matching task (Experiment 2). The results in our study indicate similar findings for errors in matching oblique lines in a perceptual (visual) matching task and in an active matching task, where subjects match the orientation of a bar to that of a moving line by arm movements. The similar pattern of errors in these two conditions might be interpreted as evidence against the hypothesis of two different pathways for "vision for perception" and "vision for action". In our study subjects had to match the orientation of a single line. In most studies on differences in "vision for perception" and "vision for action" responses were studied to stimuli in different contexts, for example for the Tichener circles illusion, where the size of the inner circle surrounded by other circles with different diameters had to be estimated. Whether or not context has an effect on differences in "vision for perception" and "vision for action" is not clear and might be a topic for further research.

The visual matching data suggested that participants did not use the final perceived orientation of the moving line to match its orientation. No evidence was found that participants used a fixed sampling time interval, since no differences matching between errors for slowly and fast moving lines were found. Instead, a small but significant effect was found of the horizontal position at which the movement started. This effect is consistent with the assumption that participants looked away from the moving line early after it started its movement, since errors in the percept of line orientation increase with the distance between the matching line and the reference line.

Although participants were instructed to match the orientation of the hidden line at the moment at which it passed through the interception point, they ended their interception movements too late. The results of a motion extrapolation experiment showed that participants could well estimate the duration of the hidden interval. The timing errors in the matching experiments might have occurred because participants focussed on correctly orienting the bar. Important is that the timing errors were not related to the size of the orientation matching errors. This means that the duration between stimulus presentation and matching did not affect the size of the matching errors. 


\section{References}

Berthoz, A. (1993). Multisensory control of movement. Oxford: Oxford University Press.

Castet, E., Lorenceau, J., Shiffrar, M., \& Bonnet, C. (1993). Perceived speed of moving lines depends on orientation, length, speed and luminance. Vision Research, 33(14), 1921-1936.

Cuijpers, R. H., Kappers, A. M. L., \& Koenderink, J. J. (2000). Large systematic deviations in visual parallellism. Perception, 29, 1467-1482.

Gentilucci, M. (2002). Object motor representation and reaching-grasping control. Neuropsychologia, 40(8), 1139-1153.

Goodale, M. A., \& Milner, D. A. (1992). Separate visual pathways for perception and action. Trends in Neurosciences, 15(1), 20-25.

Haffenden, A. M., Chif, K. C., \& Goodale, M. A. (2000). The dissociation between perception and action in the ebbinghaus illusion: Nonillusory effects of pictorial cues on the grasp. Current Biology, 11, 177181.

Haffenden, A. M., \& Goodale, M. A. (1998). The effect of pictorial illusion on prehension and perception. Journal of Cognitive Neuroscience, 10(1), 122-136.

Hermens, F., \& Gielen, S. (2003). Visual and haptic matching of perceived orientations of lines. Perception, 32(2), 235-248.

$\mathrm{Hu}$, Y., Eagleson, R., \& Goodale, M. A. (1999). The effects of delay on the kinematics of grasping. Experimental Brain Research, 126, 109-116.

Hu, Y., \& Goodale, M. A. (2000). Grasping after a delay shifts size-scaling from absolute to relative metrics. Journal of Cognitive Neuroscience, 12(5), 856-868.

Johansson, R. S., Westling, G. R., Backstrom, A., \& Flanagan, J. R. (2001). Eye-hand coordination in object manipulation. Journal of Neuroscience, 21(17), 6917-6932.

Kappers, A. M. L., \& Koenderink, J. J. (1999). Haptic perception of spation relations. Perception, 28, 781795.

Lyon, D. R., \& Waag, W. L. (1995). Time course of visual extrapolation accuracy. Acta Psychologica, 89, 239-260.

Neggers, S. F., \& Bekkering, H. (2000). Ocular gaze is anchored to the target of an ongoing pointing movement. Journal of Neurophysiology, 83, 639-651.

Neggers, S. F., \& Bekkering, H. (2001). Gaze anchoring to a pointing target is present during the entire pointing movement and is driven by a non-visual signal. Journal of Neurophysiology, 86, 961-970.

Pizlo, Z. (1994). A theory of shape constancy based on perspective invariants. Vision Research, 34(12), $1637-1658$

Rosenbaum, D. A. (1975). Perception and extrapolation of velocity and acceleration. Journal of Experimental Psychology: Human Perception and Performance, 1(4), 395-403.

Santello, M., \& Soechting, J. F. (1998). Gradual molding of the hand to object contours. Journal of Neurophysiology, 79(3), 1307-1320.

Savelsbergh, G. J. P., \& Whiting, H. T. A. (1996). Catching: A motor learning and developmental perspective. In H. Heuer, \& S. Keele (Eds.), Handbook of perception and action: Motor Skills (pp. 461497). London: Academic Presss Limited, chapter 8.

Sharp, R. H., \& Whiting, H. T. A. (1974). Exposure and occluded duration effects in a ballcatching skill. Journal of Motor Behavior, 6(3), 139-147.

Sokolov, A. N., Ehrenstein, W. H., Pavlova, M. A., \& Cavonius, C. R. (1997). Motion extrapolation and velocity transposition. Perception, 26, 875-889.

Westwood, D. A., McEachern, T., \& Roy, E. A. (2001). Delayed grasping of a Müller-Lyer figure. Experimental Brain Research, 141, 166-173.

Wiener, E. L. (1962). Motion prediction as a function of target speed and duration of presentation. Journal of Applied Psychology, 46(6), 420-424. 